\title{
Effects of a dietary excess of leucine and of the addition of leucine and 2-oxo-isocaproate on the metabolism of tryptophan and niacin in isolated rat liver cells
}

\author{
BY DAVID A. BENDER \\ Department of Biochemistry, University College and Middlesex School of Medicine, \\ University College London, Gower Street, London WC1E 6BT
}

(Received 10 June 1988-Accepted 6 January 1989)

\begin{abstract}
1. Feeding rats on a low-tryptophan, niacin-free, high-leucine diet resulted in impaired synthesis from tryptophan of the nicotinamide nucleotide coenzymes, NAD and NADP, and $\mathrm{N}^{1}$-methyl nicotinamide in isolated hepatocytes, compared with cells from animals fed on a low-tryptophan, niacin-free control diet providing an appropriate amount of leucine. This was accompanied by reduced accumulation of the tryptophan metabolites kynurenine, 3-hydroxykynurenine and xanthurenic acid.

2. With hepatocytes from animals fed on the low-tryptophan, niacin-free control diet, the addition of leucine to the incubation medium resulted in reduced synthesis of niacin from tryptophan, and a small increase in the accumulation of 3-hydroxykynurenine.

3. With hepatocytes from animals fed on the low-tryptophan, niacin-free control diet, the addition of 2-oxoisocaproate to the incubation medium resulted in increased synthesis of NAD(P) and niacin, and increased accumulation of 3-hydroxykynurenine.

4. The results suggest that a dietary excess of leucine alters the activity of the enzymes of tryptophan $\rightarrow$ niacin metabolism, or the uptake of tryptophan into the liver, in a different manner from the simple inhibition and activation seen in experiments in vitro.

5. Differences between studies in isolated hepatocytes and intact animals suggest that extra-hepatic metabolism of tryptophan, catalysed by indoleamine dioxygenase, is more important than has been believed hitherto.
\end{abstract}

Gopalan \& Srikantia (1960) proposed that a dietary excess of leucine may be a precipitating factor in the development of pellagra in people whose intake of tryptophan and niacin is otherwise apparently adequate. A number of studies in experimental animals conducted in their laboratory in Hyderabad (for review, see Belavady, 1987) and elsewhere (Yamada et al. 1979, 1983; Magboul \& Bender, 1983) have confirmed that adding leucine to diets that provide minimally adequate amounts of tryptophan can lead to depletion of tissue reserves of the nicotinamide nucleotide coenzymes NAD and NADP, and precipitate pellagra-like signs. Other studies, generally conducted under less extreme conditions, have failed to show any pellagragenic action of a dietary excess of leucine in experimental animals (Nakagawa et al. 1975; Manson \& Carpenter, 1978 $a, b$ ).

Magboul \& Bender (1983) showed that a dietary excess of leucine only affected niacin nutritional status when the animals were wholly or partially reliant on de novo synthesis of nicotinamide nucleotides from tryptophan, and not when minimally adequate amounts of preformed niacin were provided in the diet, suggesting that leucine affects tryptophan metabolism (see Fig. 1) rather than the utilization of niacin. Leucine may inhibit the de novo synthesis of NAD(P) from tryptophan by inhibiting kynureninase (EC 3.7.1.3) (Magboul \& Bender, 1983); its oxo-acid, 2-oxo-isocaproate, also inhibits kynurenine 3-hydroxylase (EC 1.14.13.9) (Magboul, 1982; Bender \& McCreanor, 1985) and giving a high-leucine diet also appears to increase the activity of picolinate carboxylase (aminocarboxymuconatesemialdehyde decarboxylase; $E C$ 4.1.1.45), thus diverting tryptophan metabolites away from NAD(P) synthesis (Ghafoorunissa \& Narasinga-Rao, 1973; Bender, 1983).

Bender (1983) investigated the effect of a dietary excess of leucine on the metabolic flux of tryptophan through discrete sectors of the oxidative pathway (see Fig. 1) in intact 


\section{Tryptophan}

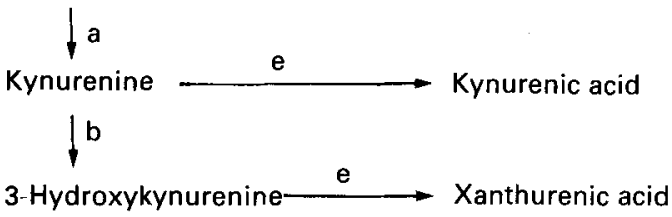

$f^{c}$

3-Hydroxyanthranilic acid

f d

Aminocarboxymuconic semialdehyde

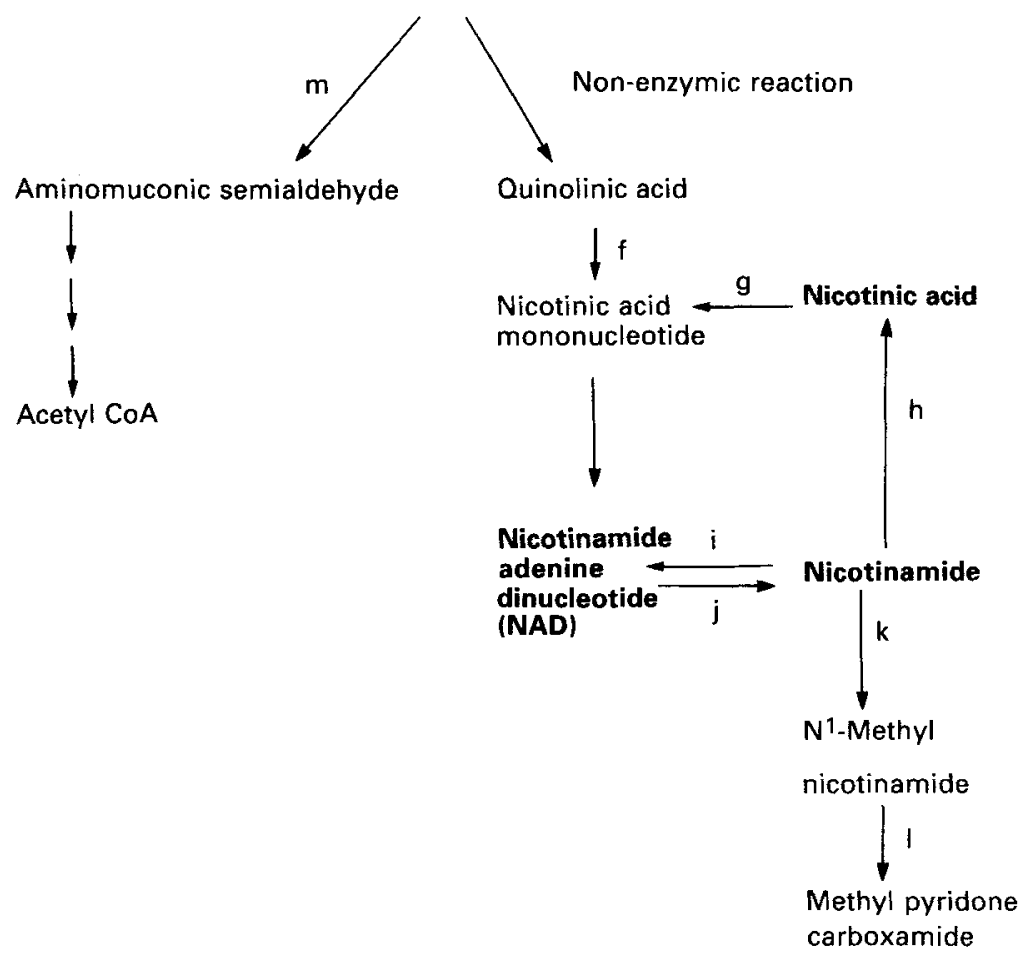

Fig. 1. The metabolism of tryptophan and nicotinamide nucleotides. a, Tryptophan 2,3-dioxygenase (EC 1.13.11.11); b, kynurenine 3-hydroxylase $(E C 1.14 .13 .9) ; \mathrm{c}$, kynureninase $(E C$ 3.7. 1.3); d, 3hydroxyanthranilate 3,4-dioxygenase $(E C 1.13 .11 .6)$; e, kynurenine aminotransferase $(E C 2.6 .1 .7) ; \mathrm{f}$, quinolinate phosphoribosyltransferase (decarboxylating) $(E C 2.4 .2 .19)$; $\mathrm{g}$, nicotinate phosphoribosyltransferase $(E C 2.4 .2 .11)$; h, nicotinamidase $(E C 3.5 .1 .19)$; i, nicotinamide phosphoribosyltransferase (EC 2.4.2.12); j, $\mathrm{NAD}^{+}$nucleosidase $\left(E C\right.$ 3.2.2.5) or $\mathrm{NAD}^{+} \mathrm{ADP}$-ribosyltransferase (EC 2.4.2.30); k, nicotinamide $N$-methyltransferase $(E C 2.1 .1 .1) ; 1$, aldehyde oxidase $(E C$ 1.2.3.1); $\mathrm{m}$, picolinate carboxylase (aminocarboxymuconate-semialdehyde decarboxylase; EC 4.1.1.45).

animals, by injecting positional isomers of $\left[{ }^{14} \mathrm{C}\right]$ tryptophan and measuring the production of ${ }^{14} \mathrm{CO}_{2}$. The results were compatible with both inhibition at the level of kynureninase or kynurenine 3-hydroxylase, or both, and increased activity of picolinate carboxylase. However, similar studies of tryptophan metabolic flux in isolated hepatocytes derived from animals maintained on a low-tryptophan, high-leucine diet showed no effect on metabolic flux through kynureninase or picolinate carboxylase (Salter et al. 1985). 
The present study was designed to investigate these differences between the apparent effects of a dietary excess of leucine on tryptophan metabolism in intact animals (Bender, 1983) and in isolated hepatocytes (Salter et al. 1985), and to investigate the possible physiological importance of the changes in enzyme activity associated with leucine that have been reported previously (Ghafoorunissa \& Narasinga-Rao, 1973; Bender, 1983; Magboul \& Bender, 1983; Bender \& McCreanor, 1985). The metabolism of tryptophan and the de novo synthesis and metabolism of nicotinamide nucleotides has been investigated in hepatocytes isolated from animals fed on a low-tryptophan, high-leucine diet, and in hepatocytes isolated from animals fed on a low-tryptophan diet, incubated in the presence of leucine or its oxo-acid, 2-oxo-isocaproate.

\section{METHODS}

Male Wistar rats bred in University College London were used (this is the same sub-strain as that used in previous studies, see Bender, 1983; Magboul \& Bender, 1983; Bender \& McCreanor, 1985). At $21 \mathrm{~d}$ after birth, animals were weaned onto the low-tryptophan, niacin-free diet described previously by Magboul \& Bender (1983), a modification of the maize-gelatine-sucrose diet described by Carter et al. (1977). In the high-leucine diet $15 \mathrm{~g}$ L-leucine $/ \mathrm{kg}$ diet was added at the expense of gelatine.

After the animals had been receiving the diets for 7-8 weeks, they were anaesthetized with $150 \mathrm{mg}$ pentobarbitone $/ \mathrm{kg}$ body-weight, and hepatocytes were prepared by portalcaval perfusion of the liver with collagenase $(E C 3.4 .24 .3)$ as described previously (Bender \& Olufunwa, 1988), a modification of the methods described by Elliott et al. (1976) and Romero \& Viña (1983), except that once recirculation of the perfusion medium was commenced, it was brought to $2.5 \mathrm{mmol}$ calcium ions/l. This modification permits the preparation of a good yield of metabolically active hepatocytes in a shorter time, and using a lower concentration of collagenase, than the procedure used previously, in which the perfusion medium was Ca-free throughout (Seglen, 1976).

After washing, the cells were suspended in ice-cold Krebs-Ringer phosphate-bicarbonate buffer containing $5 \mathrm{mmol}$ glucose $/ 1$, to give $8-10 \mathrm{mg}$ dry weight of cells $/ \mathrm{ml}$. In each incubation, $1 \mathrm{ml}$ of this cell suspension was mixed with $7 \mathrm{ml}$ Krebs-Ringer phosphate-bicarbonate buffer containing $(\mathrm{mmol} / \mathrm{l}): 5$ glucose, 4.5 lactate, 0.5 pyruvate, 5 glutamine (as described by Salter \& Pogson, 1985); $1 \mathrm{ml}$ of a solution of tryptophan (over a range from 0.031 to $8 \mathrm{mmol} / \mathrm{l}$ ), as in previous studies (Bender \& Olufunwa, 1988), and $1 \mathrm{ml}$ of a solution of leucine $(8 \mathrm{mmol} / \mathrm{l}), 2$-oxo-isocaproate $(8 \mathrm{mmol} / \mathrm{l})$ in Krebs-Ringer phosphate-bicarbonate buffer containing $5 \mathrm{mmol}$ glucose/l, or buffer alone, in siliconized $25 \mathrm{ml}$ conical flasks, which were sealed with rubber stoppers (Suba-seals). The cells were incubated at $37^{\circ}$ for $30 \mathrm{~min}$ with gentle shaking ( $100 \mathrm{rev} . / \mathrm{min}$ ) and were gassed throughout the incubation with oxygen-carbon dioxide $(95: 5, \mathrm{v} / \mathrm{v})$. All incubations were performed in duplicate on the hepatocytes prepared from each animal. In studies of the effects of a dietary excess of leucine, portions of the cell suspension prepared from each animal were incubated with all nine concentrations of tryptophan in any one experiment. The studies on the effect of adding leucine or 2-oxo-isocaproate to the incubation medium were performed only on hepatocytes isolated from animals fed on the control diet. In these experiments cells from each animal were incubated with all nine concentrations of tryptophan, both with and without the addition of either leucine or 2-oxo-isocaproate.

At the end of the incubation, flasks were transferred to an ice-bath. A $5 \mathrm{ml}$ sample from each was centrifuged at $2000 \mathrm{~g}$ for $10 \mathrm{~min}$. The resultant cell pellet was used for determination of nicotinamide nucleotides (total NAD and NADP, oxidized and reduced forms) by the modification of the fluorimetric methods of Kaplan et al. (1951) and Lowry 
et al. (1961) that has been described previously (Bender et al. 1982; Bender \& Olufunwa, 1988). The supernatant fraction (incubation medium) was used for the determination of $\mathrm{N}^{1}$-methyl nicotinamide by the small-scale modification of the fluorimetric method of Carpenter \& Kodicek (1950) that has been described previously (Bender, 1980) and total niacin (nicotinamide plus nicotinic acid) by a modification of the cyanide adduct method of Carlson (1966), after conversion of nicotinamide to nicotinic acid using a crude preparation of nicotinamide deamidase from Micrococcus lysodeikticus (nicotinamidase; EC 3.5.1.19; Sigma (London) Chemical Co. Ltd, Poole, Dorset) as described previously (Bender \& Olufunwa, 1988). This procedure was adopted since nicotinamide and nicotinic acid yield cyanide adducts with the same absorption spectrum but very different molar extinction coefficients; the absorbance of the nicotinic acid adduct is about 100 -fold greater than that of the nicotinamide adduct. In samples from some incubations, niacin was determined before and after deamidation of nicotinamide to permit calculation of the concentrations of each vitamer separately.

The remaining $5 \mathrm{ml}$ from each incubation was placed in a boiling water-bath for $5 \mathrm{~min}$, then centrifuged at $2000 \mathrm{~g}$ for $10 \mathrm{~min}$. The supernatant fraction (incubation medium and cell contents) was used for determination of kynurenine, by the diazotization procedure of Joseph \& Risby (1975); 3-hydroxykynurenine, by the fluorimetric method of Watanabe et al. (1970); and xanthurenic acid by the alkali fluorimetric method of Satoh \& Price (1958). The amount of sample available, and the concentration of analytes, did not permit ionexchange chromatography to separate xanthurenic and kynurenic acids from interfering materials, as described by Satoh \& Price (1958). While it was possible to determine xanthurenic acid simply by adding $14 \mathrm{~mol}$ sodium hydroxide/l, the addition of sulphuric acid resulted in the formation of variable amounts of coloured materials that interfered in the fluorimetric determination of kynurenic acid. Kynurenic acid was, therefore, not determined.

Statistical analysis was performed by two-way analysis of variance on the mean values from duplicate incubations, using the Instat statistics package (Statistical Service Centre, University of Reading, Reading, Berks).

\section{RESULTS}

The effects of maintaining rats on the low-tryptophan, high-leucine diet for 7-8 weeks are shown in Fig. 2. There was a small but significant impairment of the synthesis of NAD(P) from tryptophan, and a considerably more marked reduction in the synthesis of $\mathrm{N}^{1}$-methyl nicotinamide in hepatocytes from the leucine-fed animals. This was accompanied by a reduction in the accumulation of kynurenine, 3-hydroxykynurenine and xanthurenic acid.

In samples from the incubations performed with $8 \mathrm{mmol}$ tryptophan/l, niacin was determined before and after the enzymic conversion of nicotinamide to nicotinic acid. In hepatocytes from animals receiving the low-tryptophan control diet, 40 (SEM 1.9) \% of the total niacin was nicotinamide, whereas in hepatocytes from animals receiving the lowtryptophan, high-leucine diet, 63 (SEM 2.9$) \%$ of the total was nicotinamide $(P<0.001 ; t$ test).

Fig. 3 shows the effect of adding $8 \mathrm{mmol}$ leucine/l to the incubation medium on the metabolism of tryptophan in hepatocytes isolated from animals fed on the low-tryptophan control diet. Leucine had no effect on the synthesis of NAD(P) or $\mathrm{N}^{1}$-methyl nicotinamide, although there was a significant reduction in the amount of niacin (nicotinic acid + nicotinamide) released. The addition of leucine to the incubation medium resulted in a small increase in the accumulation of 3-hydroxykynurenine, but had no effect on kynurenine or xanthurenic acid. 

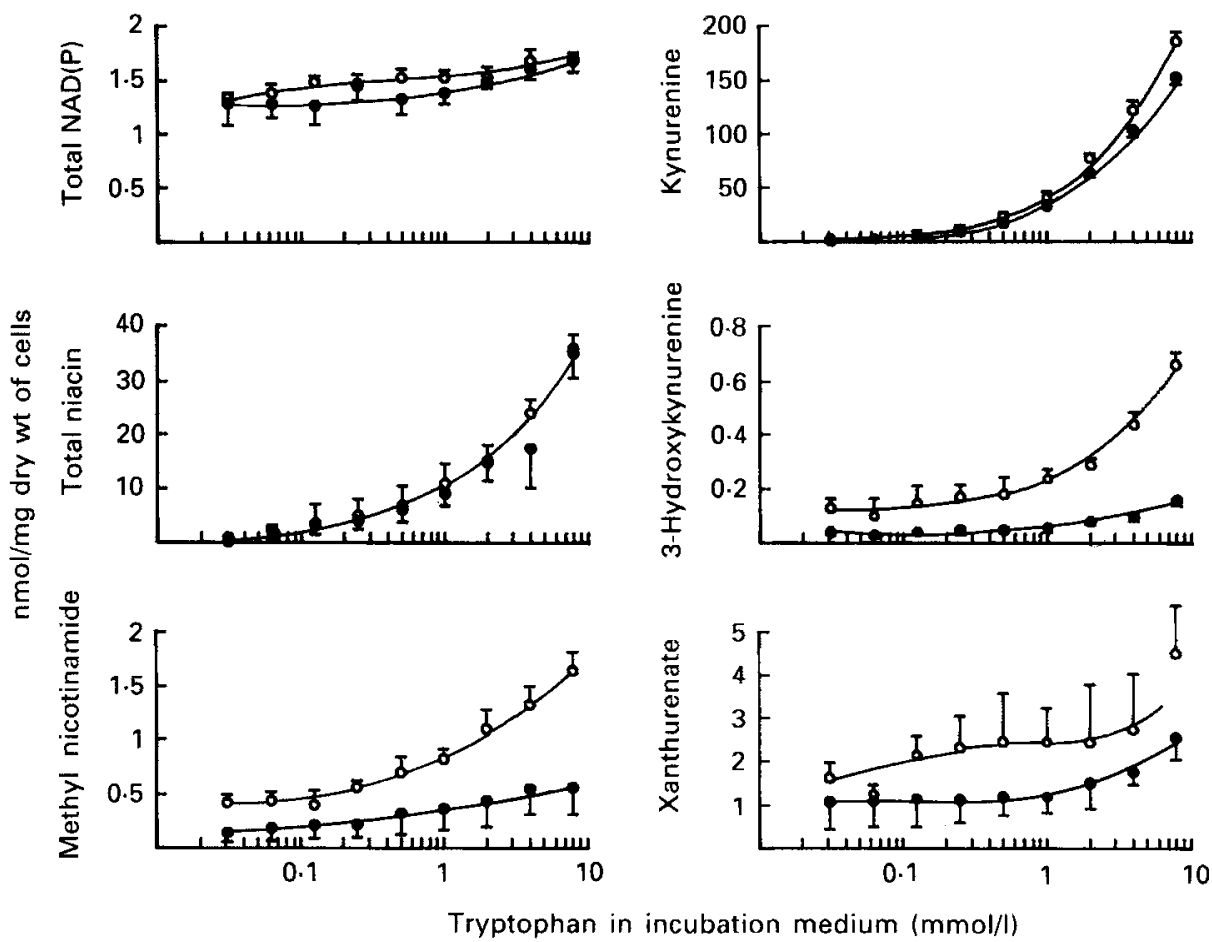

Fig. 2. The metabolism of tryptophan and nicotinamide nucleotide coenzymes in hepatocytes isolated from rats fed on a low-tryptophan diet, with (O) or without $(O)$ the addition of $15 \mathrm{~g}$ leucine/ $\mathrm{kg}$ diet. Points represent means, with their standard errors represented by vertical bars, for duplicate incubations of hepatocytes from each of three animals receiving each diet; cells from each animal were incubated with all nine concentrations of tryptophan. For details of procedures, see pp. 631-632.

Analysis of variance

Variance attributable to:...

Tryptophan concentration (8 df)

Statistical
significance:

Analyte:

NAD (P)

Niacin

$\mathrm{N}^{\mathbf{1}}$-Methyl nicotinamide

Kynurenine

3-Hydroxykynurenine

Xanthurenic acid

NS, not significant.

$\begin{array}{rr}9.8 & 0.0020 \\ 109.2 & <0.0001 \\ 4.1 & 0.0311 \\ 112.5 & <0.0001 \\ 2.5 & 0.1083 \\ 2.7 & 0.0908\end{array}$

Diet (l df)

\begin{tabular}{rc}
\hline & $\begin{array}{c}\text { Statistical } \\
\text { significance: }\end{array}$ \\
$F$ & $P$ \\
11.2 & 0.0101 \\
2.7 & NS \\
25.1 & 0.0010 \\
5.7 & 0.0441 \\
17.2 & 0.0032 \\
6.5 & 0.0342
\end{tabular}

Fig. 4 shows the effect of adding $8 \mathrm{mmol} 2$-oxo-isocaproate/ 1 to the incubation medium on the metabolism of tryptophan in hepatocytes isolated from animals fed on the lowtryptophan control diet. This resulted in a significant increase in the synthesis of NAD(P) and niacin from tryptophan, with no effect on the synthesis of $\mathrm{N}^{1}$-methyl nicotinamide. This was accompanied by a small decrease in kynurenine accumulation and a small increase in the accumulation of 3-hydroxykynurenine. 

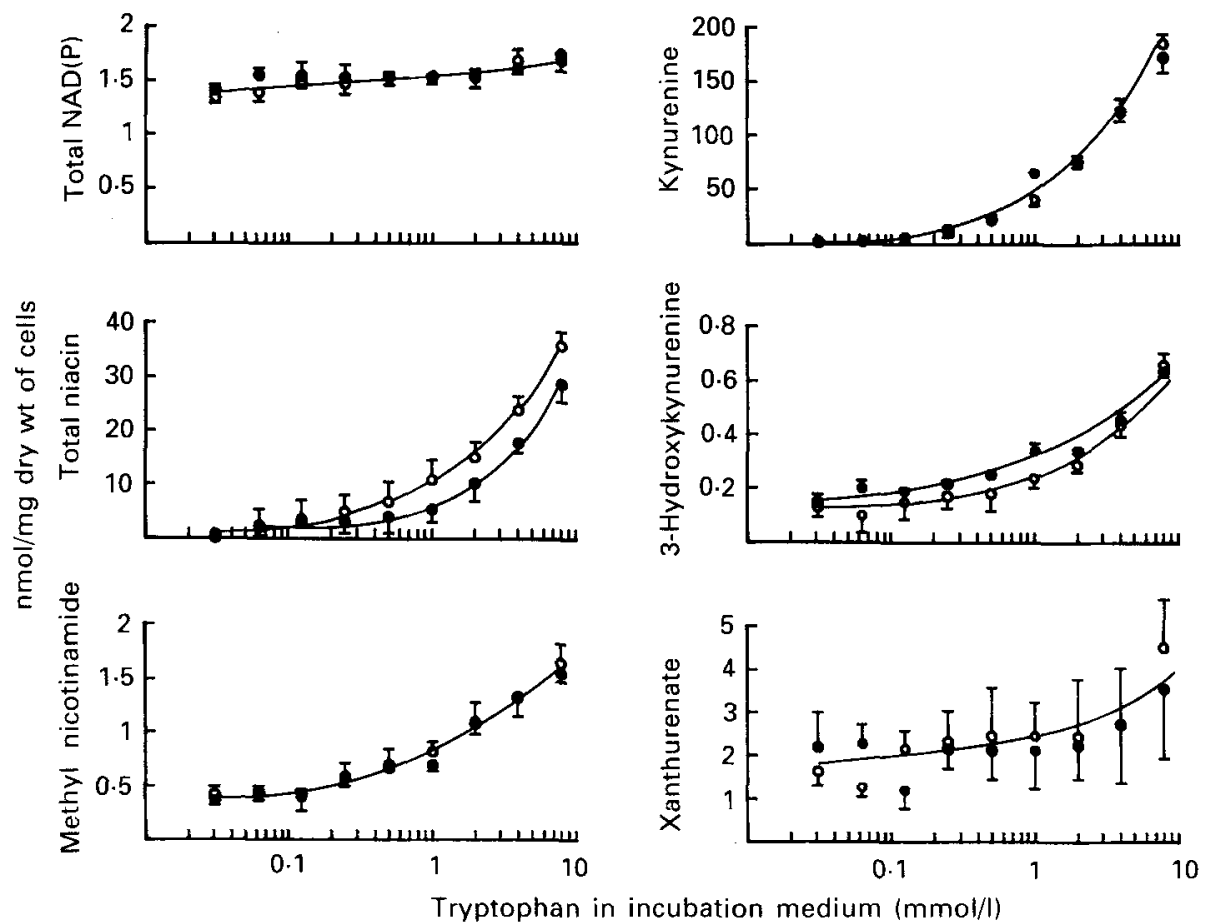

Fig. 3. The effects of adding leucine on the metabolism of tryptophan and nicotinamide nucleotide coenzymes in hepatocytes isolated from rats fed on a low-tryptophan, niacin-free diet. $(O)$, Control incubations; (O), incubations in the presence of $8 \mathrm{mmol} \mathrm{L}$-leucine/l. Points represent means, with their standard errors represented by vertical bars, for duplicate incubations of hepatocytes from each of three animals; cells from each animal were incubated with and without leucine, at all nine concentrations of tryptophan. For details of procedures, see pp. 631-632.

Analysis of variance

Variance attributable to:...

Tryptophan concentration $(8 \mathrm{df})$

$\begin{array}{cc}\text { Statistical } \\ \text { significance: } \\ F & P\end{array}$

\begin{tabular}{rc}
\multicolumn{2}{c}{ Leucine $(\mathrm{I} \mathrm{d})$} \\
\hline & $\begin{array}{c}\text { Statistical } \\
\text { significance: }\end{array}$ \\
$F$ & $P$ \\
& \\
2.9 & $\mathrm{NS}$ \\
9.9 & 0.0137 \\
2.5 & $\mathrm{NS}$ \\
0.3 & $\mathrm{NS}$ \\
13.5 & 0.0065 \\
0.5 & $\mathrm{NS}$
\end{tabular}

$\begin{array}{rr}9.2 & 0.0025 \\ 51.8 & <0.0001 \\ 268.8 & <0.0001 \\ 165.9 & <0.0001 \\ 74.6 & <0.0001 \\ 4.9 & 0.0187\end{array}$

NS, not significant. 

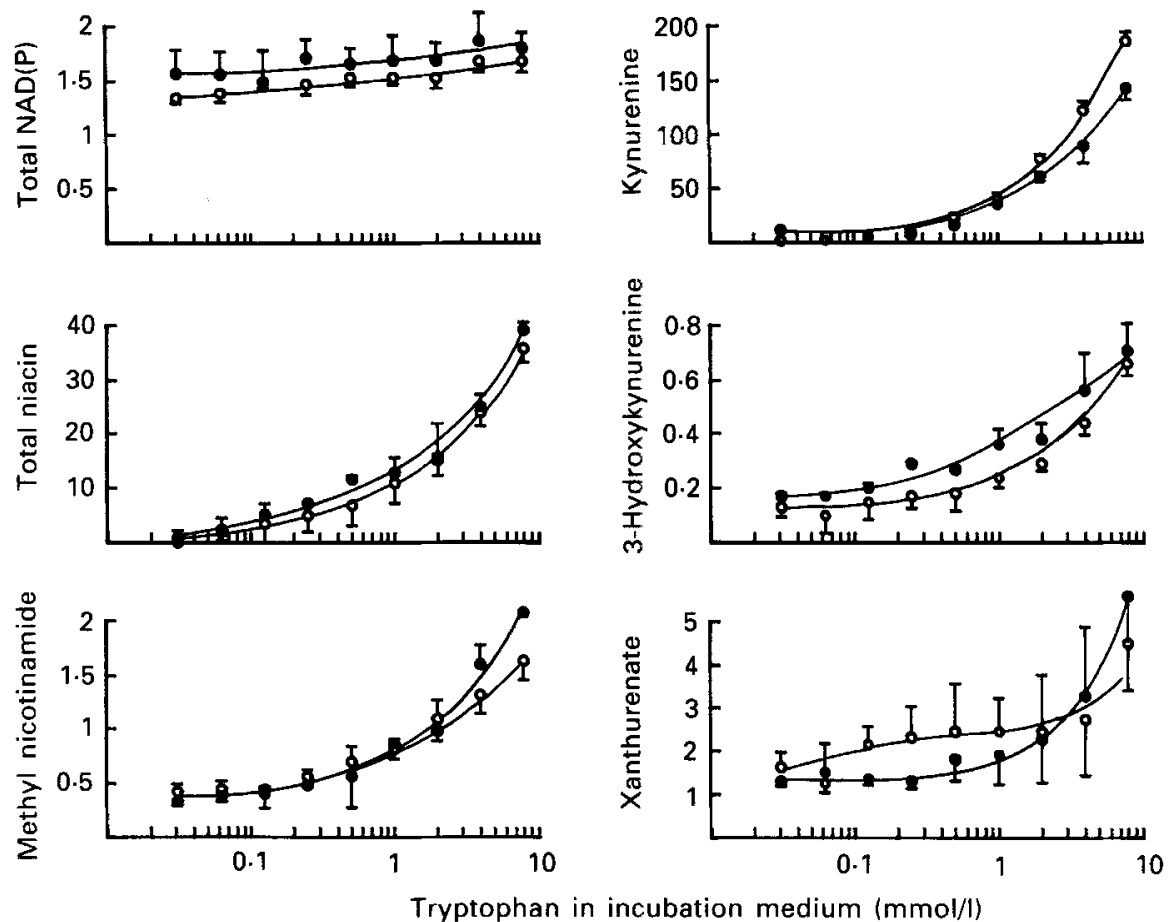

Fig. 4. The effects of adding 2-oxo-isocaproate on the metabolism of tryptophan and nicotinamide nucleotide coenzymes in hepatocytes isolated from rats fed on a low-tryptophan, niacin-free diet. ( $O$ ), Control incubations; $(O)$, incubations in the presence of $8 \mathrm{mmol} 2$-oxo-isocaproate/l. Points represent means, with their standard errors represented by vertical bars, for duplicate incubations of hepatocytes from each of three animals; cells from each animal were incubated with and without 2-oxo-isocaproate, at all nine concentrations of tryptophan. For details of procedures, see pp. 631-632.

Variance attributable to:...

\begin{tabular}{c}
$\begin{array}{c}\text { Tryptophan concentration } \\
(8 \mathrm{df})\end{array}$ \\
\hline \\
$\begin{array}{c}\text { Statistical } \\
\text { significance } \\
P\end{array}$
\end{tabular}

Analyte:

NAD (P)

Niacin

$\mathrm{N}^{1}$-Methyl nicotinamide

Kynurenine

$9 \cdot 8$

3-Hydroxykynurenine

Xanthurenic acid

283.9

27.7

$52 \cdot 7$

131.5

$11 \cdot 1$
2-Oxo-isocaproate (1 df)

$\begin{array}{rc} & \begin{array}{r}\text { Statistical } \\ \text { significance } \\ F\end{array} \\ & P \\ 43.3 & 0.0002 \\ 15.5 & 0.0043 \\ 0.3 & \text { NS } \\ 5.2 & 0.0520 \\ 61.0 & <0.0001 \\ 0.6 & \text { NS }\end{array}$

NS, not significant. 


\section{DISCUSSION}

These results confirm previous observations that under extreme conditions, when animals are largely or wholly reliant on a minimally adequate intake of tryptophan, with little or no preformed niacin, the addition of an excess of leucine to the diet inhibits the oxidative metabolism of tryptophan (Bender, 1983) and results in significantly lower tissue concentrations of the nicotinamide nucleotide coenzymes (NAD and NADP) (Magboul \& Bender, 1983). Thus leucine may indeed be a precipitating factor in the development of pellagra, as proposed by Gopalan \& Srikantia (1960).

Rats fed on the low-tryptophan, niacin-free, high-leucine diet both consume less food and grow more slowly than those maintained on an isonitrogenous diet providing an appropriate amount of leucine (Magboul \& Bender, 1983). It is not known whether the impairment of growth is the result of reduced food intake (and hence presumably an effect on appetite) or whether it reflects a more direct toxic effect of the dietary excess of leucine. However, a dietary excess of leucine does result in a significant reduction in the amount of nicotinamide nucleotides per $\mathrm{g}$ of tissue (Magboul \& Bender, 1983). In the present study, animals were allowed access to food $a d$ lib., and, therefore, it is not possible to exclude effects of food deprivation, as opposed to specific effects of the dietary excess of leucine.

Rats fed on a low-tryptophan, high-leucine diet excrete significantly more kynurenine than do animals fed on a low-tryptophan control diet, and leucine is an inhibitor of partially purified preparations of kynureninase (Magboul \& Bender, 1983). Studies of the metabolism of [methylene- ${ }^{14} \mathrm{C}$ ] tryptophan in intact animals also indicate inhibition of tryptophan metabolism at the level of kynureninase (Bender, 1983). The present results do not show this effect. Hepatocytes from animals fed on the high-leucine diet accumulated less kynurenine than did those from control animals (see Fig. 2), and the addition of leucine to the incubation medium had no effect on the accumulation of kynurenine during incubation (see Fig. 3). This is despite the fact that the final concentration of leucine in the incubations, $8 \mathrm{mmol} / 1$, was considerably higher than the normal plasma concentration of $0.1 \mathrm{mmol} / 1$ reported by Hutson \& Harper (1981). It is also higher than the concentration of $1 \mathrm{mmol} / 1$ reported by Yamada et al. (1983) in rats fed on a diet providing $0.5 \mathrm{~g}$ excess leucine $/ \mathrm{kg}$ diet. This high concentration of leucine was used because it is the level at which inhibition of partially purified enzyme preparations has been observed in previous studies (Magboul, 1982; Magboul \& Bender, 1983).

These studies were performed using a wide range of concentrations of tryptophan, from 0.031 to $8 \mathrm{mmol} / 1$. The normal plasma concentration of tryptophan in the strain of rat used in these studies is $0.1 \mathrm{mmol} / 1$; in response to a variety of chronic drug treatments, plasma concentrations of tryptophan between 0.05 and $0.150 \mathrm{mmol} / \mathrm{l}$ have been observed (Bender \& Cockcroft, 1977). However, the dose of tryptophan used in the tryptophan load test is between 30 and $75 \mathrm{mg} / \mathrm{kg}$ body-weight (Coursin, 1964; Allegri et al. 1978); this might be expected to result in a (transient) increase in plasma tryptophan of between 0.7 and 1.8 mmol/1. Despite the relatively high upper concentrations of tryptophan used in the incubations, there was relatively little increase in the hepatocyte concentration of the nicotinamide nucleotide coenzymes, NAD and NAD(P). Rather, there was a considerable increase in the production of niacin and $\mathrm{N}^{\mathrm{l}}$-methyl nicotinamide. This is in agreement with results reported previously: a high dietary intake of tryptophan results in only a modest increase in liver NAD(P), with a more considerable increase in urinary excretion of $\mathrm{N}^{1}$ methyl nicotinamide and its onward metabolite, methyl pyridone carboxamide (Bender et al. 1982; McCreanor \& Bender, 1986). Also, incubation of hepatocytes isolated from rats fed on animal-house stock diet (Diet 86; A. Dixon \& Sons, Ware, Herts) with the same concentrations of tryptophan as used in the present study showed little increase in NAD(P), 
and a considerable increase in the production of free niacin, $\mathbf{N}^{1}$-methyl nicotinamide and methyl pyridone carboxamide (Bender \& Olufunwa, 1988). These results suggest that, while there is increased synthesis of NAD(P) in the presence of large amounts of tryptophan, most of this is hydrolysed to release nicotinamide, which is then either released or undergoes methylation and onward oxidation. Enzyme kinetic and other studies suggest that hydrolysis of NAD(P) is an important factor in controlling liver concentrations of the coenzymes (McCreanor \& Bender, 1983), and may well help to prevent an undesirable accumulation at times of high synthesis from tryptophan.

Based on estimations of the activity of partially purified enzyme preparations, Knox (1953) suggested that kynureninase might be a secondary rate-limiting enzyme in tryptophan oxidative metabolism. A more rigorous approach to metabolic regulation involves the calculation of control coefficients of individual steps in the pathway (for review, see Kacser \& Porteous, 1987). The control coefficient of an individual step is that proportion of the total control of metabolic flux which can be attributed to the step under consideration; the sum of all the control coefficients for a given pathway is, by definition, 1.0. Salter et al. (1986) have determined the control coefficient for kynureninase to be < 0.004 . They suggest that control of metabolic flux, as opposed to calculation based on unidirectional rates of enzyme action, resides mainly in tryptophan 2,3-dioxygenase ( $E C$ 1.13 .11 .11 ; control coefficient 0.75 ) and the initial step of uptake of tryptophan into the cells (control coefficient $0 \cdot 25$ ). They showed that when the activity of tryptophan 2,3dioxygenase is maximally increased, transport becomes the most important factor controlling the rate of tryptophan oxidative metabolism, with a control coefficient of 0.75 . Since leucine and tryptophan are substrates for the same carrier system (Pardridge, 1977), inhibition of tryptophan uptake, and hence impairment of tryptophan metabolism, might be expected in the presence of leucine. Salter et al. (1985) demonstrated such competition in incubations of hepatocytes in the presence of both leucine and tryptophan. The results of the present study are in agreement with the conclusion that the activity of kynureninase is not a significant controlling factor in the metabolism of tryptophan in isolated hepatocytes.

The results in Fig. 2 show that both the synthesis of $\mathrm{NAD}(\mathrm{P})$ and $\mathrm{N}^{1}$-methyl nicotinamide, and the accumulation of tryptophan metabolites (kynurenine, 3-hydroxykynurenine and xanthurenic acid) were reduced in hepatocytes from animals fed on the high-leucine diet. It is unlikely that this can be attributed to simple competition between leucine and tryptophan for uptake into the cells, since the cells were prepared by a process of perfusion and extensive washing, during which extracellular amino acids, including both leucine and those other large neutral amino acids which compete with tryptophan for the L-system carrier mechanism (Pardridge, 1977) would be removed. Furthermore, addition of leucine to the incubation medium does not have the same effects on tryptophan metabolism (see Fig. 3). It is, therefore, likely that giving the high-leucine diet for a period of 7-8 weeks affects the activity of either the cell membrane amino acid transport system or tryptophan 2,3-dioxygenase.

In intact animals, leucine undergoes transamination in muscle, and a significant proportion of the resultant 2-oxo-isocaproate is transported to the liver for further metabolism; little leucine is normally transaminated in the liver (Haymond \& Miles, 1981; Christensen, 1982). However, there seems to be tight control over the release of 2-oxoisocaproate into the circulation from muscle (Hutson \& Harper, 1981). As can be seen from Fig. 4, adding 2-oxo-isocaproate to the incubation medium had a significant effect on tryptophan-niacin metabolism, resulting in increases in $\mathrm{NAD}(\mathrm{P})$, niacin, $\mathrm{N}^{1}$-methyl nicotinamide, kynurenine and 3-hydroxykynurenine. Such an effect might result from either increased uptake of tryptophan into the cells in the presence of 2-oxo-isocaproate, 
or from increased activity of tryptophan 2,3-dioxygenase. Magboul (1982) showed that the addition of $7.6 \mathrm{mmol}$ 2-oxo-isocaproate/l to a partially purified preparation of tryptophan 2,3-dioxygenase increased the activity of the enzyme from 1.7 (SEM 0.20) to 4.2 (SEM 0.27) nmol kynurenine formed/min per $\mathrm{g}$ liver. This is compatible with the high control coefficient for tryptophan 2,3-dioxygenase in isolated hepatocytes reported by Salter et al. (1986). It is probable that if circulating concentrations of 2-oxo-isocaproate rose to a high enough level to increase the activity of tryptophan 2,3-dioxygenase, this might mask any inhibition of tryptophan metabolism by leucine. Such an effect may help to explain the discrepancies between those experiments which have demonstrated an effect of a dietary excess of leucine on tryptophan-niacin metabolism (Yamada et al. 1979; Magboul \& Bender, 1983; Belavady, 1987) and those which have failed to demonstrate any effect (Nakagawa et al. 1975; Manson \& Carpenter, $1978 a, b$ ); the balance between inhibition of kynureninase by leucine and activation of tryptophan 2,3-dioxygenase by its oxo-acid, 2oxo-isocaproate, may be critically important.

2-Oxo-isocaproate inhibits partially purified preparations of kynurenine hydroxylase (Magboul, 1982; Bender \& McCreanor, 1985). However, addition of 2-oxo-isocaproate to the incubation medium does not seem to inhibit kynurenine hydroxylase in isolated hepatocytes. It is possible that any such inhibition is masked by the increased metabolic flux resulting from increased activity of tryptophan 2,3-dioxygenase, as discussed previously. However, 2-oxo-isocaproate is a relatively weak inhibitor of kynurenine hydroxylase, with a $K_{i}$ (concentration of inhibitor giving half maximal inhibition) of 63 (SD 18) $\mathrm{mmol} / 1$ (Bender \& McCreanor, 1985), while liver concentrations of 2-oxo-isocaproate do not normally rise above $5-10 \mu \mathrm{mol} / 1$ (Hutson \& Harper, 1981), and the control coefficient of kynurenine hydroxylase is only 0.037 (Salter et al. 1986). This suggests that inhibition of the hydroxylase by 2-oxo-isocaproate is unlikely to be a significant factor affecting the synthesis of $\mathrm{NAD}(\mathrm{P})$ from tryptophan.

The activity of a partially purified preparation of tryptophan 2,3-dioxygenase is also increased by the addition of leucine; Magboul \& Bender (1983) showed an increase in activity from 1.7 (SEM 0.20 ) to 3.0 (SEM 0.20 ) nmol kynurenine formed/min per g liver on incubation in the presence of $7.6 \mathrm{mmol} / 1$ leucine. In the present study, this effect may well have been masked by the competition between tryptophan and leucine for uptake into the cells. Such opposing effects would explain the relatively small effects of leucine added to the incubation medium on the synthesis of tryptophan and niacin metabolites shown in Fig. 3 .

The present results, and those reported by Salter et al. (1985) in isolated hepatocytes, contrast markedly with those in intact animals, where giving a high-leucine diet results in increased excretion of kynurenine (Magboul \& Bender, 1983) and impairment of tryptophan metabolism through kynureninase (Bender, 1983). It is likely that this discrepancy reflects extra-hepatic metabolism of tryptophan, presumably catalysed by indoleamine dioxygenase, which catalyses essentially the same reaction as tryptophan 2,3dioxygenase, and results in the formation of kynurenine. The metabolic fate of kynurenine produced in extra-hepatic tissues is unknown. These results suggest that indoleamine dioxygenase may be more important in whole-body tryptophan and niacin metabolism than has hitherto been considered.

The technical assistance of Julius Kamau (Department of Biochemistry, University of Nairobi, Kenya), Deepan Shah, Violet Trim and Francis B. Zotor (Department of Biochemistry, Ghana Medical School, Accra, Ghana) is gratefully acknowledged. J. K. and F.B.Z. were recipients of British Council Technical Assistance Training Awards. 


\section{REFERENCES}

Allegri, G., Costa, C. \& de Antoni, A. (1978). A further contribution to the choice of the dose for tryptophan load test. Acta Vitaminologica Enzymologica (Milan) 32, $163-166$.

Belavady, B. (1987). Amino acid imbalance and pellagra. In Progress in Tryptophan and Serotonin Research, vol. 2 pp. 153-158 [D. A. Bender, M. H. Joseph, W. Kochen and H. Steinhart, editors]. Berlin: Walter de Gruyter.

Bender, D. A. (1980). Effects of benserazide, carbidopa and isoniazid administration on tryptophan nicotinamide nucleotide metabolism in the rat. Biochemical Pharmacology 29, 2099-2104.

Bender, D. A. (1983). Effects of a dietary excess of leucine on the metabolism of tryptophan in the rat: a mechanism for the pellagragenic action of leucine. British Journal of Nutrition 50, 25-32.

Bender, D. A. \& Cockcroft, P. M. (1977). Increase in brain tryptophan and 5-hydroxytryptamine on administration of phenothiazines to rats. Biochemical Society Transactions 5, 155-157.

Bender, D. A. \& McCreanor, G. M. (1985). Kynurenine hydroxylase: a potential rate-limiting enzyme in tryptophan metabolism. Biochemical Society Transactions 13, 441-443.

Bender, D. A., Magboul, B. I. \& Wynick, D. (1982). Possible mechanisms of regulation of the utilization of dietary tryptophan, nicotinamide and nicotinic acid as precursors of nicotinamide nucleotides in the rat. British Journal of Nutrition 48, 119-127.

Bender, D. A. \& Olufunwa, R. (1988). Utilization of tryptophan, nicotinamide and nicotinic acid as precursors for nicotinamide nucleotide synthesis in isolated rat liver cells. British Journal of Nutrition 59, 279-287.

Carlson, L. A. (1966). Determination of free nicotinic acid in blood plasma. Clinica Chimica Acta 13, 349-351.

Carpenter, K.\& Kodicek, E. (1950). The fluorimetric estimation of $\mathrm{N}^{1}$-methyl nicotinamide and its differentiation from coenzyme I. Biochemical Journal 46, 421-426.

Carter, E. G., Hurrell, R. F. \& Carpenter, K. J. (1977). A rat growth assay for the potency of foods as a source of niacin. Proceedings of the Nutrition Society 36, 107A.

Christensen, H. N. (1982). Inter-organ amino acid metabolism. Physiological Reviews 62, 1193-1233.

Coursin, D. B. (1964). Recommendations for standardization of the tryptophan load test. American Journal of Clinical Nutrition 14, 56-61.

Elliott, K. R. F., Ash, R., Pogson, C. I., Smith, S. A. \& Crisp, D. M. (1976). In Use of Isolated Liver Cells and Kidney Tubules in Metabolic Studies, pp. 139-143 [J. M. Tager, H. D. Soling and J. R. Williamson, editors]. Amsterdam: North Holland.

Ghafoorunissa \& Narasinga-Rao, B. S. (1973). Effect of leucine on the tryptophan-niacin pathway in rat liver and kidney. Biochemical Journal 134, 425-430.

Gopalan, C. \& Srikantia, S. G. (1960). Leucine and pellagra. Lancet i, 954-957.

Haymond, M. W. \& Miles, J. M. (1981). Branched chain amino acids as a major source of alanine nitrogen in man. Diabetes 31, 86-89.

Hutson, S. M. \& Harper, A. E. (1981). Blood and tissue branched chain amino and 2-oxo acid concentrations: effect of diet, starvation and disease. American Journal of Clinical Nutrition 34, 173-183.

Joseph, M. H. \& Risby, D. (1975). The determination of kynurenine in plasma. Clinica Chimica Acta 63, $197-204$.

Kacser, H. \& Porteous, J. W. (1987). Control of metabolism: what do we have to measure? Trends in Biochemical Sciences 12, 5-14.

Kaplan, N. O., Colowick, S. P. \& Barnes, C. C. (1951). Effect of alkali on diphosphopyridine nucleotides. Journal of Biological Chemistry 191, 461-472.

Knox, W.E. (1953). The relation of liver kynureninase to tryptophan metabolism in pyridoxine deficiency. Biochemical Journal 53, 379-385.

Lowry, O. H., Passoneau, J. V. \& Rock, M. K. (1961). The stability of pyridine nucleotides. Journal of Biological Chemistry 236, 2756-2759.

McCreanor, G. M. \& Bender, D. A. (1983). The role of catabolism in controlling tissue concentrations of nicotinamide nucleotide coenzymes. Biochimica et Biophysica Acta 759, 222-228.

McCreanor, G. M. \& Bender, D. A. (1986). The metabolism of high intakes of tryptophan, nicotinamide and nicotinic acid in the rat. British Journal of Nutrition 56, $577-586$.

Magboul, B. I. (1982). Studies on experimental niacin depletion in the rat. PhD Thesis, University of London.

Magboul, B. I. \& Bender, D. A. (1983). Effects of a dietary excess of leucine on the synthesis of nicotinamide nucleotides in the rat. British Journal of Nutrition 49, 321-329.

Manson, J. A. \& Carpenter, K. J. (1978 a). The effect of a high level of dietary leucine on the niacin status of chicks and rats. Journal of Nutrition 108, 1883-1888.

Manson, J. A. \& Carpenter, K. J. (1978b). The effect of a high level of dietary leucine on the niacin status of dogs. Journal of Nutrition 108, 1888-1898.

Nakagawa, T., Onguri, S., Sasaki, A., Kajimoto, M., Sasaki, M. \& Takahashi, T. (1975). Effects of excess of leucine and valine deficiency on tryptophan and niacin metabolism in humans. Journal of Nutrition 105 , $1241-1252$

Pardridge, W. M. (1977). Kinetics of competitive inhibition of neutral amino acid transport across the blood--brain barrier. Journal of Neurochemistry 28, 103-108.

Romero, F. J. \& Viña, J. (1983). A simple procedure for the preparation of isolated liver cells. Biochemical Education 11, 135-137. 
Salter, M., Bender, D. A. \& Pogson, C. I. (1985). Leucine and tryptophan metabolism in rats. Biochemical Journal 225, 277-281.

Salter, M., Knowles, R. G. \& Pogson, C. I. (1986). Quantification of the importance of individual steps in the control of aromatic amino acid metabolism. Biochemical Journal 234, 635-647.

Salter, M. \& Pogson, C. I. (1985). The role of tryptophan 2,3-dioxygenase in the hormonal control of tryptophan metabolism in isolated rat liver cells. Biochemical Journal 229, 499-504.

Satoh, K. \& Price, J. M. (1958). Fluorimetric determination of kynurenic acid and xanthurenic acid in human urine. Journal of Biological Chemistry 230, 781-789.

Seglen, P. O. (1976). Preparation of isolated rat liver cells. Methods in Cell Biology 13, $29-83$.

Watanabe, M., Watanabe, Y. \& Okada, M. (1970). A new fluorimetric method for the determination of 3 . hydroxykynurenine. Clinica Chimica Acta $27,461-466$.

Yamada, O., Shin, M., Sano, K. \& Umezawa, C. (1979). Effect of a dietary excess of leucine on nicotinamide nucleotides in rat liver. International Journal for Vitamin and Nutrition Research 49, 376-385.

Yamada, O., Shin, M., Sano, K. \& Umezawa, C. (1983). Effect of leucine and $\alpha$-keto-isocaproic acid on NAD biosynthesis from tryptophan or nicotinic acid in the isolated rat liver cells. International Journal for Vitamin and Nutrition Research 53, 184-191. 